\title{
Neuropsychiatric Symptoms and Syndromes in a Large Cohort of Newly Diagnosed, Untreated Patients With Alzheimer Disease
}

\author{
Gianfranco Spalletta, M.D., Ph.D., Massimo Musicco, M.D., \\ Alesandro Padovani, M.D., Ph.D., Luca Rozzini, M.D., Roberta Perri, M.D., Ph.D., \\ Lucia Fadda, Psy.D., Vincenzo Canonico, M.D., Alberto Trequattrini, M.D., \\ Carla Pettenati, M.D., Carlo Caltagirone, M.D., Katie Palmer, Ph.D.
}

\begin{abstract}
Objectives: Neuropsychiatric symptoms are common in patients with Alzheimer disease (AD). Treatment for both AD and psychiatric disturbances may affect the clinical observed pattern and comorbidity. The authors aimed to identify whether particular neuropsychiatric syndromes occur in untreated patients with $A D$, establish the severity of syndromes, and investigate the relationship between specific neuropsychiatric syndromes and $A D$ disease severity. Design: Cross-sectional, multicenter, clinical study. Participants: A total of 1,015 newly diagnosed, untreated outpatients with AD from five Italian memory clinics were consecutively enrolled in the study from January 2003 to December 2005. Measurements: All patients underwent thorough examination by clinical neurologists/geriatricians, including neuropsychiatric symptom evaluation with the Neuropsychiatric Inventory. Results: Factor analysis revealed five distinct neuropsychiatric syndromes: the apathetic syndrome (as unique syndrome) was the most frequent, followed by affective syndrome (anxiety and depression), psychomotor (agitation, irritability, and aberrant motor behavior), psychotic (delusions and hallucinations), and manic (disinbibition and euphoria) syndromes. More than three quarters of patients with AD presented with one or more of the syndromes $(N=790,77.8 \%)$, and more than half exbibited clinically significant severity of symptoms $(N=603,59.4 \%)$. With the exception of the affective one, all syndromes showed an increased occurrence with increasing severity of dementia. Conclusions: The authors' study supports the use of a syndrome approach for neuropsychiatric evaluation in patients with AD. Individual neuropsychiatric symptoms can be reclassified into five distinct psychiatric syndromes. Clinicians should incorporate a thorough psychiatric and neurologic examination of patients with AD and consider therapeutic strategies that focus on psychiatric syndromes, rather than specific individual symptoms. (Am J Geriatr Psychiatry 2010; 18:1026-1035)
\end{abstract}

\footnotetext{
Received October 15, 2009; revised December 4, 2009; accepted December 9, 2009. From the Fondazione Santa Lucia, Instituto di Ricovero e Cura a Carettere Scientifico (IRCCS), Rome (GS, MM, RP, LF, CC, KP); Consiglio Nazionale delle Ricerche (CNR), Institute of Biomedical Technologies, Milan, Italy (MM); Department of Neurological Sciences, University of Brescia, Brescia, Italy (AP); Unità di Valutazione, Alzheimer Cattedra di Geriatria Università degli Studi "Federico II" Napoli (VC); ASL Città di Castello, Perugia, Italy (AT); Alzheimer Center Hospital of Passirana di Rho (Milan), Milan, Italy (CP); and Department of Neuroscience, Tor Vergata University, Rome, Italy (CC). Send correspondence and reprint requests to Katie Palmer, Ph.D., IRCCS Fondazione Santa Lucia, Via Ardeatina 306, 00179 Roma, Italy. e-mail: k.palmer@hsantalucia.it

(C) 2010 American Association for Geriatric Psychiatry
} 
Key Words: Psychiatric syndromes, apathy, depression, cognitive impairment, behavioral and psychological symptoms of dementia, BPSD

$\mathrm{T}$ he core symptom of Alzheimer disease (AD), the most common primary degenerative dementia, is memory impairment, which is accompanied by deficits in at least one other cognitive domain. However, both in AD and other primary degenerative dementias, neuropsychiatric symptoms are common. ${ }^{1}$ Almost all patients with AD exhibit neuropsychiatric symptoms at some point during the course of the disease. ${ }^{2-4}$

Difficulties arise when characterizing neuropsychiatric symptoms of patients with AD because they are heterogeneous and unpredictable. ${ }^{5}$ These symptoms range from mood alterations (depression, anxiety, euphoria, and disinhibition) to psychotic manifestations (delusions and hallucinations), various symptoms with motor manifestations (apathy, agitation, wandering, and aggressiveness) and sleep or appetite symptoms. ${ }^{2}$ Some authors have suggested that this heterogeneity is due to neuropsychiatric symptoms resulting from complex interactions of the pathologic process with the premorbid specific personality and the environmental exposures or life experiences of the patient. ${ }^{6,7}$

One approach to better understand the psychiatric manifestations of AD is to evaluate whether specific symptoms occur in association. Rather than viewing individual symptoms separately, a limited number of syndromes featuring associated symptoms are identified. The potential advantages of this approach are that neuropsychiatric syndromes might be less heterogeneous and unpredictable and might be more similar to primary psychiatric disorders, thus allowing potentially fruitful analogies in terms of pathogenesis, prognostic evaluation and treatment. ${ }^{8} \mathrm{How}-$ ever, studies have reported that the behavioral and psychological syndromes associated with AD are not completely consistent with the disorders seen in patients affected by primary psychiatric diseases. ${ }^{9-12}$ For example, although many studies report the existence of a psychotic syndrome characterized by the concomitant occurrence of delusions and hallucinations, $^{13,14}$ these two specific psychotic symptoms are also accompanied by symptoms of a different nature, such as anxiety ${ }^{10}$ or agitation and irritability. ${ }^{11}$ Current knowledge is limited by the fact that many studies have been conducted on patients who are undergoing treatment for either AD or neuropsychiatric problems, which can alter the pattern and severity of psychiatric disturbances. Indeed, a European consortium study ${ }^{12}$ reported an influence of acetyl cholinesterase inhibitors on apathy and psychosis in AD, and it has been shown that donepezil treatment reduces psychotic symptoms, ${ }^{15}$ and prazosin reduces global neuropsychiatric symptom severity and frequency. ${ }^{16}$

The aim of this study was to identify neuropsychiatric syndromes in a large cross-sectional cohort of newly diagnosed patients with $\mathrm{AD}$, who were not treated either for AD or neuropsychiatric phenomena. Specifically, we aimed to: a) investigate whether specific neuropsychiatric syndromes can be characterized in patients with $\mathrm{AD}, \mathrm{b}$ ) establish the occurrence and severity of neuropsychiatric syndromes, and c) investigate the relationship between specific neuropsychiatric syndromes and progression of the neurodegenerative process, measured as the severity of dementia.

\section{METHODS}

\section{Study Sample}

Patients newly diagnosed as having probable AD, who were consecutively seen in five Italian outpatient memory clinics, were included in the study. The diagnosis of AD was made according to National Institute of Neurological and Communicative Diseases and Stroke-Alzheimer's Disease and Related Disorders Association (NINCDS-ARDA) ${ }^{17}$ by trained clinical neurologists and geriatricians. All patients were free from treatment with either psychotropic drugs or acetylcholinesterase inhibitors and had sufficient vision and hearing abilities for compliance with testing procedures (eyeglasses and/or hearing aids permissible). 
We excluded patients who did not have a "reliable" caregiver defined as a person who had contact with the patient twice a week, including at least one personal visit. Exclusion criteria were established through examination of the patient and review of medical records, and interviews with the caregiver, and included i) major medical illnesses, i.e., diabetes not stabilized, obstructive pulmonary disease or asthma, hematologic/ oncologic disorders, B12 or folate deficiency as evidenced by blood concentrations below the lower normal limit, pernicious anemia, clinically significant and unstable active gastrointestinal, renal, hepatic, endocrine or cardiovascular system diseases, newly treated hypothyroidism, liver function tests (alanine transaminasi [ALT] aspartate aminotransferase [AST]) greater than three times the upper normal limit, creatinine concentrations $>150 \mu \mathrm{mol} / \mathrm{L}$; ii) presence of psychiatric disorders with onset before AD (e.g., schizophrenia and major depression); iii) presence of neurologic disorders (e.g., stroke, Parkinson disease, seizure disorder, and head injury with loss of consciousness within the past year); iv) known or sus- pected history of alcoholism or drug abuse; v) patients undergoing treatment with psychotropic drugs or acetylcholinesterase inhibitors; vi) computed tomography or magnetic resonance imaging evidence of focal brain parenchymal abnormalities; and vii) nursing home patients or institutionalization within last 3 months.

Based on the inclusion criteria, 1,015 subjects were consecutively enrolled in the study from January 2003 to December 2005. The sociodemographic, clinical, cognitive, functional, and behavioral characteristics are summarized in Table 1 . The study sample included patients with $\mathrm{AD}$ with a mean Mini-Mental State Examination (MMSE) ${ }^{18}$ score of 18.3 at first diagnosis of $\mathrm{AD}$. This is similar to previous reports on the mean scores for patients with $\mathrm{AD}$ at first diagnosis in clinical settings. ${ }^{19,20}$ Less than one fifth of patients already had severe dementia, despite the fact that we included only patients with a new diagnosis of AD. This reflects the clinical picture of dementia diagnoses today, because there is a large variation in the time between the onset of first symptoms and first physician consultation, with

TABLE 1. Total and Gender-Specific Demographic and Clinical Characteristics of the Study Sample

\begin{tabular}{|c|c|c|c|c|c|c|c|c|c|}
\hline & \multicolumn{2}{|c|}{$\begin{array}{c}\text { Men } \\
(n=292) \\
\end{array}$} & \multicolumn{2}{|c|}{$\begin{array}{c}\text { Women } \\
(n=723)\end{array}$} & \multicolumn{2}{|c|}{$\begin{array}{c}\text { Total } \\
(\mathrm{n}=\mathbf{1 , 0 1 5}) \\
\end{array}$} & \multicolumn{3}{|c|}{$\begin{array}{c}\text { Differences Between Men } \\
\text { and Women }\end{array}$} \\
\hline & $\mathbf{n}$ & Percent & $\mathbf{n}$ & Percent & $\mathbf{n}$ & Percent & $\chi^{2}$ & $d f$ & $\mathbf{p}$ \\
\hline \multicolumn{10}{|l|}{ Age at disease onset, years } \\
\hline$>65$ & 219 & 75.0 & 616 & 85.2 & 835 & 82.3 & & & \\
\hline$\leq 65$ & 73 & 25.0 & 107 & 14.8 & 180 & 17.7 & 14.835 & 1 & 0.000 \\
\hline \multicolumn{10}{|l|}{ Disease severity } \\
\hline Mild & 137 & 46.9 & 246 & 34.0 & 383 & 37.7 & & & \\
\hline Moderate & 118 & 40.4 & 336 & 46.5 & 454 & 44.7 & & & \\
\hline \multirow[t]{2}{*}{ Severe } & 37 & 12.7 & 141 & 19.5 & 178 & 17.5 & 16.406 & 2 & 0.000 \\
\hline & Mean & SD & Mean & SD & Mean & SD & t Test & $d f$ & $\mathbf{p}$ \\
\hline Age, years (45-96) & 73.3 & 0.5 & 75.1 & 0.3 & 74.6 & 0.2 & & & \\
\hline Education, years $(0-19)$ & 7.6 & 0.2 & 5.7 & 0.1 & 6.2 & 0.1 & -3.499 & 1,013 & 0.000 \\
\hline \multicolumn{10}{|l|}{ Disease duration yrs (0- } \\
\hline 24) & 2.8 & 0.1 & 2.7 & 0.1 & 2.7 & 0.1 & 7.862 & 7.862 & 0.697 \\
\hline MMSE $(0-30)$ & 19.2 & 0.3 & 18.0 & 0.2 & 18.3 & 0.2 & 0.390 & 0.390 & 0.001 \\
\hline Instrumental ADL (0-8) & 3.5 & 0.1 & 4.4 & 0.1 & 4.2 & 0.1 & -5.625 & 1,013 & 0.000 \\
\hline \multicolumn{10}{|l|}{ NPI items $(0-12)$} \\
\hline Delusions & 1.0 & 0.1 & 1.2 & 0.1 & 1.1 & 0.1 & -1.131 & 1,013 & 0.258 \\
\hline Hallucinations & 0.5 & 0.1 & 0.7 & 0.1 & 0.6 & 0.1 & -1.768 & 1,013 & 0.077 \\
\hline Agitation & 1.5 & 0.1 & 1.7 & 0.1 & 1.6 & 0.1 & -0.869 & 1,013 & 0.385 \\
\hline Depression & 2.2 & 0.2 & 2.8 & 0.1 & 2.7 & 0.1 & -3.263 & 1,013 & 0.001 \\
\hline Anxiety & 1.6 & 0.1 & 2.3 & 0.1 & 2.1 & 0.1 & -3.695 & 1,013 & 0.000 \\
\hline Euphoria & 0.3 & 0.1 & 0.4 & 0.0 & 0.4 & 0.0 & 1.899 & 1,013 & 0.058 \\
\hline Apathy & 3.1 & 0.2 & 2.9 & 0.1 & 3.0 & 0.1 & 1.020 & 1,013 & 0.308 \\
\hline Disinhibition & 0.7 & 0.1 & 0.6 & 0.1 & 0.6 & 0.1 & 0.081 & 1,013 & 0.936 \\
\hline Irritability & 1.6 & 0.2 & 1.6 & 0.1 & 1.6 & 0.1 & 0.096 & 1,013 & 0.924 \\
\hline Aberrant motor & & & & & & & & & \\
\hline behavior & 1.1 & 0.1 & 1.3 & 0.1 & 1.3 & 0.1 & -1.523 & 1,013 & 0.128 \\
\hline Total NPI score $(0-81)$ & 13.6 & 0.7 & 15.7 & 0.5 & 15.1 & 0.4 & -2.300 & 1,013 & 0.022 \\
\hline
\end{tabular}


some patients seeking medical care only when the disease is already at a severe stage. ${ }^{19,21-23}$

\section{Cognitive, Functional, and Behavioral Evaluations}

The clinicians who made the $\mathrm{AD}$ diagnoses, assessed all cognitive, functional, and behavioral characteristics of the patients, including an interview with next of kin and caregivers. Education was assessed as the number of years of formal education. Age at onset was defined as age at the onset of cognitive symptoms severe enough to compromise patient functioning and was assessed through an interview with the caregiver.

Overall cognitive decline was assessed with the MMSE. ${ }^{18}$ Instrumental activities of daily living (IADL) were assessed with the IADL scale. ${ }^{24}$

Behavioral symptoms were measured with the Neuropsychiatric Inventory (NPI)-10 items. ${ }^{25}$ The NPI is a valid and reliable inventory assessing 10 neuropsychiatric dimensions in patients with dementia or other neurologic disorders. The investigated dimensions/ symptoms are delusions, hallucinations, agitation, depression, anxiety, euphoria, apathy, disinhibition, irritability, and aberrant motor behavior. An informant rates the frequency $(0-3)$ and severity $(0-4)$ of each of these dimensions and the multiplication of the two scores is used as final score, which ranges from 0 to 12 for each dimension, with a maximum total score of 120 in the 10-item version.

\section{Ethical Considerations}

The ethical committee of the participating centers approved the protocol of the study. The nature and purposes of this study were presented to the patients and explained to their responsible caregivers and/or legal guardians. Written informed consent was obtained from patients or patients' representatives and caregivers before beginning the screening activities.

\section{Statistical Analyses}

First, baseline characteristics of the study sample were calculated by gender, including disease severity, sociodemographics, cognitive and functional status, and NPI performance. $\chi^{2}$ and Student's $t$ tests were used to assess differences between categorical and continuous variables, respectively. Second, we calculated the correlation index between MMSE and NPI total score among all patients, with Pearson correlation test. Third, to identify the principal components of neuropsychiatric symptoms and examine the syndrome associations of neuropsychiatric symptoms occurring together, we carried out a factor analysis on the 10 NPI symptoms within the total group of patients with AD. The neuropsychiatric symptoms were first analyzed with a principal component factor analysis with varimax rotation. This analysis was performed to reduce the 10 NPI symptoms to a limited number of groups of symptoms (factors) occurring in association. Each factor was defined by the total variance explained, and by the symptoms that were grouped together. A symptom entered on the respective factor when the loading was $>0.5$ and when its presence within the factor was logically consistent. Following this, we categorized each patient on the basis of the presence of one or more of the syndromes disclosed by the factor analysis. The severity of the syndromes was calculated for each patient as follows: "no syndrome" when patients scored 0 on at least one of the symptoms in the syndrome, "mild syndrome" when patients had NPI score between 1 and 3 on every symptom in the syndrome, and "clinically significant syndrome" when patients scored $\geq 1$ on all symptoms in the syndrome with at least one symptom score $\geq 4 .^{26}$

Patients were further divided according to the severity of the dementia measured by the MMSE score: mild AD (MMSE $\geq 18$ ), moderate AD (MMSE = 11-17), and severe AD (MMSE $\leq 10)$. The proportion of patients with clinically significant neuropsychiatric syndromes was then calculated according to dementia severity. Finally, we performed logistic regression analyses to calculate the odds of having a clinically significant syndrome according to i) disease severity, with mild AD as the reference category and ii) IADL score. Odds ratios (ORs) and 95\% confidence intervals (CIs) were calculated with logistic regression models. For all analyses, statistical significance was set at the 0.05 level.

\section{RESULTS}

Demographic and clinical characteristics of the patients are shown in Table 1. Women were more likely 
to have a disease onset $>65$ years and had higher age and IADL score. Men were more likely to have mild disease severity, higher age, education, and MMSE. Women had a higher mean NPI score for depression, anxiety, and total NPI score.

The correlation index between MMSE and NPI total score was $-0.185(\mathrm{p}=0.002) ; 3.4 \%$ of the variance in the NPI score was explained by MMSE (squared correlation index $=-0.185^{2}=0.034$ ). Although the correlation is statistically significant, this result shows that $<4 \%$ of the entire NPI variance is explained by differences in global cognitive functioning. This suggests that at a group level, patients with AD with more severe cognitive deficits also have more severe neuropsychiatric symptoms, but that at the individual level, the severity of symptoms is poorly predicted by the severity of cognitive deficits.

Table 2 shows the results of the factor analysis of 10 NPI symptoms in 1,015 patients with AD. The analysis revealed five principal components (factors) among the 10 NPI symptoms. These five factors explained $>76 \%$ of the variability observed in the 10 symptomatologic dimensions of NPI. The first factor revealed a "psychomotor syndrome," comprised of agitation, irritability, and aberrant motor behavior. The second factor was strictly and uniquely related to delusions and hallucinations, clearly indicating the existence of a "psychotic syndrome." The third component revealed an "affective syndrome" comprised of anxiety and depression. The fourth component was characterized by euphoria and disinhibition, suggesting the existence of a "manic syndrome." Finally, the fifth factor was related uniquely to apathy, revealing an "apathetic syn-

TABLE 2. Factor Analysis on the 10 Items of NPI. Five Factors Explain $76.0 \%$ of Total Variance

\begin{tabular}{lrrrrr}
\hline & \multicolumn{5}{c}{ Factor and Eigen values } \\
\cline { 2 - 6 } & \multicolumn{1}{c}{$\mathbf{1}$} & $\mathbf{2}$ & \multicolumn{1}{c}{$\mathbf{3}$} & \multicolumn{1}{c}{$\mathbf{4}$} & \multicolumn{1}{c}{$\mathbf{5}$} \\
\hline Delusions & 0.245 & $\mathbf{0 . 8 4 0}$ & 0.013 & 0.148 & 0.078 \\
Hallucinations & 0.064 & $\mathbf{0 . 8 9 7}$ & 0.072 & 0.090 & -0.038 \\
Agitation & $\mathbf{0 . 6 9 2}$ & 0.395 & 0.195 & 0.066 & -0.025 \\
Depression & -0.031 & 0.132 & $\mathbf{0 . 8 2 5}$ & -0.049 & 0.258 \\
Anxiety & 0.223 & -0.024 & $\mathbf{0 . 8 4 1}$ & 0.122 & -0.075 \\
Euphoria & -0.029 & 0.152 & 0.039 & $\mathbf{0 . 8 8 7}$ & 0.017 \\
Apathy & 0.072 & 0.019 & 0.151 & 0.078 & $\mathbf{0 . 9 3 1}$ \\
Disinhibition & 0.375 & 0.091 & 0.049 & $\mathbf{0 . 6 8 4}$ & 0.128 \\
Irritability & $\mathbf{0 . 8 4 0}$ & 0.111 & 0.149 & 0.057 & 0.017 \\
Aberrant motor & $\mathbf{0 . 6 1 3}$ & -0.088 & -0.104 & 0.338 & 0.403 \\
\multicolumn{1}{c}{ behavior } & & & & & \\
\hline
\end{tabular}

drome." It is noteworthy that all the symptoms contributed positively to the severity of only one of the five factors, suggesting that each NPI symptom is independently characteristic of only one of the syndromes.

The severity of the syndromes was calculated for each patient including 1) no, 2) mild, and 3) clinically significant syndrome. Table 3 shows the occurrence of syndromes and severity according to sex. Most patients presented one or more of the five syndromes $(\mathrm{N}=790,77.8 \%)$, and the majority had syndromes severe enough to be clinically significant $(N=603$, $59.4 \%$ ). The apathetic syndrome was the most frequent followed by affective (depression and anxiety) and psychomotor (irritability, aberrant motor behavior, and agitation) syndromes. The fourth

TABLE 3. Occurrence and Severity of Psychiatric Syndromes by Gender

\begin{tabular}{lcccc}
\hline Syndrome & Men & $\begin{array}{c}\text { Women } \\
\text { N (\%) }\end{array}$ & $\begin{array}{c}\text { Total } \\
\text { n (\%) }\end{array}$ & p \\
\hline Any syndrome & n (\%) & & & \\
$\quad$ No & $69(23.6)$ & $156(21.6)$ & $225(22.2)$ & \\
Mild & $60(20.5)$ & $127(17.6)$ & $187(18.4)$ & \\
Clinically significant & $163(55.8)$ & $440(60.9)$ & $603(59.4)$ & $0.319^{\mathrm{b}}$ \\
Psychotic & & & & \\
No & $262(89.7)$ & $632(87.4)$ & $894(88.1)$ & \\
Mild & $8(2.7)$ & $24(3.3)$ & $32(3.2)$ & \\
Clinically significant & $22(7.5)$ & $67(9.3)$ & $89(8.8)$ & $0.589^{\mathrm{c}}$ \\
Affective & & & & \\
No & $186(63.7)$ & $399(55.2)$ & $585(57.6)$ & \\
Mild & $39(13.4)$ & $94(13.0)$ & $133(13.1)$ & \\
Clinically significant & $67(22.9)$ & $230(31.8)$ & $297(29.3)$ & $0.016^{\mathrm{d}}$ \\
Manic & & & & \\
No & $279(95.5)$ & $658(91.0)$ & $937(92.3)$ & \\
Mild & $6(2.1)$ & $21(2.9)$ & $27(2.7)$ & \\
Clinically significant & $7(2.4)$ & $44(6.1)$ & $51(5.0)$ & $0.036^{\mathrm{e}}$ \\
Psychomotor & & & & \\
No & $255(87.3)$ & $620(85.7)$ & $875(86.2)$ & \\
Mild & $3(1)$ & $21(2.9)$ & $24(2.4)$ & \\
Clinically significant & $34(11.6)$ & $82(11.3)$ & $116(11.4)$ & $0.204^{\mathrm{f}}$ \\
Apathetic & & & & \\
No & $105(36.0)$ & $287(39.7)$ & $392(38.6)$ & \\
Mild & $78(26.7)$ & $157(21.7)$ & $235(23.2)$ & \\
Clinically significant & $109(37.3)$ & $279(38.6)$ & $388(38.2)$ & $0.214^{\mathrm{g}}$ \\
& & & &
\end{tabular}

\footnotetext{
${ }^{\mathrm{a}}$ Syndrome severity: no syndrome, score $=0$ on at least one of the symptoms in the syndrome; mild syndrome, NPI score 1-3 on every symptom in the syndrome; clinically significant syndrome, NPI score $\geq 1$ on all symptoms in the syndrome with at least one symptom score $\geq 4$.

${ }^{\mathrm{b}} \chi^{2}$ difference between men and women: $\chi^{2}=2.287, d f=2$.

${ }^{c} \chi^{2}$ difference between men and women: $\chi^{2}=1.060, d f=2$.

${ }^{\mathrm{d}} \chi^{2}$ difference between men and women: $\chi^{2}=8.223, d f=2$

${ }^{\mathrm{e}} \chi^{2}$ difference between men and women: $\chi^{2}=6.660, d f=2$.

${ }^{\mathrm{f}} \chi^{2}$ difference between men and women: $\chi^{2}=3.176, d f=2$.

${ }^{\mathrm{g}} \chi^{2}$ difference between men and women: $\chi^{2}=3.082, d f=2$.
} 
most common syndrome was psychotic (delusions and hallucinations) and finally the manic syndrome (euphoria and disinhibition).

Clinically significant syndromes occurred more frequently than mild ones (Table 3). For example, almost a third of patients had a clinically significant affective syndrome $(29.3 \%, \mathrm{~N}=297)$, whereas only $13.1 \%(\mathrm{~N}=133)$ presented with a mild severity of the syndrome. This pattern occurred for all five syndromes. Gender differences were observed only in the affective and manic syndromes that were more frequent in women.

Table 4 shows the occurrence of clinically significant syndromes according to the severity of dementia. All the syndromes with the exception of the affective syndrome showed a clear increase of occurrence with increasing severity of the cognitive symptoms of dementia (Table 4). For example, patients with moderate AD had a twofold odds of having the psychomotor syndrome compared with patients with mild $\mathrm{AD}$, and the odds increased to 4.2 for patients with severe AD. However, there was no increased odds of having the affective syndrome in patients with moderate $\mathrm{AD}$ or severe $\mathrm{AD}$ compared with patients with mild AD. Similarly, lower IADL performance was also associated with all of the syndromes except the affective one $(\mathrm{OR}=1.0,95 \% \mathrm{CI}=$ $0.9-1.03$, Wald $=0.67, d f=1, p=0.414$ ). The odds of having the psychotic syndrome increased by $40 \%$ with each IADL point decrease $(\mathrm{OR}=1.4,95 \% \mathrm{CI}=$ 1.3-1.5, Wald $=37.847, d f=1, p=0.000)$, and a similar patter was seen for manic $(\mathrm{OR}=1.2,95 \%$ $\mathrm{CI}=1.1-1.4$, Wald $=13.161, d f=1, \mathrm{p}=0.000)$, psychomotor $(\mathrm{OR}=1.3,95 \% \mathrm{CI}=1.2-1.4$, Wald $=$ 32.457, $d f=1, \mathrm{p}=0.000)$, and apathetic syndromes $(\mathrm{OR}=1.2,95 \% \mathrm{CI}=1.1-1.2, \mathrm{Wald}=30.541, \mathrm{df}=1$, $\mathrm{p}=0.000)$.

\section{DISCUSSION}

The current study investigated the presence of neuropsychiatric syndromes in patients with $\mathrm{AD}$. We identified distinct associations of NPI symptoms depicting five syndromes; the most common were an apathetic and an affective syndrome. Syndromes tended to occur at a clinically significant level. There

TABLE 4. Occurrence and Association Between Clinically Significant Syndromes and AD Severity

\begin{tabular}{|c|c|c|c|c|c|}
\hline & \multirow[b]{2}{*}{$\begin{array}{l}\text { No/Mild } \\
\text { Syndrome n (\%) }\end{array}$} & \multirow{2}{*}{$\begin{array}{c}\text { Clinically } \\
\text { Significant } \\
\text { Syndrome n }(\%)\end{array}$} & \multicolumn{3}{|c|}{$\begin{array}{c}\text { Odds of Having a Clinically Significant } \\
\text { Syndrome }\end{array}$} \\
\hline & & & $\begin{array}{l}\text { OR }(95 \% \\
\text { CI })\end{array}$ & Wald & $d f$ \\
\hline \multicolumn{6}{|l|}{ Psychotic } \\
\hline Mild AD & $576(94.4)$ & $34(5.6)$ & 1.0 (reference) & & \\
\hline Moderate AD & $281(87.0)$ & $42(13.0)$ & $1.9(1.1-3.2)$ & 5.459 & 1 \\
\hline Severe AD & $69(84.1)$ & $13(15.9)$ & $2.6(1.4-4.8)$ & 8.793 & 1 \\
\hline \multicolumn{6}{|l|}{ Affective } \\
\hline Mild AD & $433(71.0)$ & 177 (29.0) & 1.0 (reference) & & \\
\hline Moderate AD & $228(70.6)$ & $177(29.0)$ & $0.9(0.7-1.2)$ & 0.721 & 1 \\
\hline Severe AD & 57 (69.5) & $25(30.5)$ & $0.8(0.5-1.2)$ & 1.408 & 1 \\
\hline \multicolumn{6}{|l|}{ Manic } \\
\hline Mild AD & $592(97.0)$ & $18(3.0)$ & 1.0 (reference) & & \\
\hline Moderate AD & $298(92.3)$ & $25(7.7)$ & $2.1(1.0-4.4)$ & 3.666 & 1 \\
\hline Severe AD & $74(90.2)$ & $8(9.8)$ & $3.9(1.8-8.8)$ & 11.204 & 1 \\
\hline \multicolumn{6}{|l|}{ Psychomotor } \\
\hline Mild AD & $563(92.3)$ & $47(7.7)$ & 1.0 (reference) & & \\
\hline Moderate AD & $276(85.4)$ & $47(14.6)$ & $2.0(1.2-3.3)$ & 7.059 & 1 \\
\hline Severe AD & $60(73.2)$ & $22(26.8)$ & $4.2(2.4-7.2)$ & 26.620 & 1 \\
\hline \multicolumn{6}{|l|}{ Apathetic } \\
\hline Mild AD & 396 (64.9) & $214(35.1)$ & 1.0 (reference) & & \\
\hline Moderate AD & $197(61.0)$ & $126(39.0)$ & $1.2(0.9-1.6)$ & 1.367 & 1 \\
\hline Severe AD & $34(41.5)$ & $48(58.5)$ & $1.7(1.2-2.5)$ & 8.596 & 1 \\
\hline
\end{tabular}

Syndrome severity: no syndrome, score $=0$ on at least one of the symptoms in the syndrome; mild syndrome: NPI score $1-3$ on every symptom in the syndrome; clinically significant syndrome: NPI score $\geq 1$ on all symptoms in the syndrome with at least one symptom score $\geq 4$. 
was an increased occurrence of all psychiatric syndromes with increasing severity of $\mathrm{AD}$, with the exception of the affective syndrome. The patients included in this study were newly diagnosed and none of them were treated either for AD or for controlling neuropsychiatric disturbances. Thus, the pattern of neuropsychiatric symptoms in this cohort represents the natural occurrence of these manifestations in persons with AD.

The analysis of principal components showed that neuropsychiatric symptoms in persons with dementia can be reduced to five groups of symptoms characterizing five logically consistent syndromes. This grouping fitted well to our data and could explain $>76 \%$ of the total variance of symptom occurrence. Previous studies $^{8,9,11,12,27,28}$ identified a pattern of neuropsychiatric syndromes mainly in agreement with our findings. The most notable difference of our study compared with others ${ }^{9,27}$ was the distinct apathetic syndrome. For example, the study of Hollingworth et al. did not identify separate affective and apathetic syndromes but instead reported a general "mood disorder" containing depression, apathy, and anxiety. Proitsi et al. reported that apathy loaded both on a "behavioral discontrol" factor and on a general "mood disorder." Apathy symptoms did not load high on the "affective" component in our factor analysis but presented as an individual component. Furthermore, our findings showed that patients with the affective and apathetic syndrome had different characteristics; women were more likely to have the affective syndrome, and the occurrence did not increase with $\mathrm{AD}$ disease severity. This raises the question of why there is this discrepancy concerning apathy between ours and other studies? Our patients were free from pharmacologic treatments, had a lower mean age at examination, and had a much shorter mean disease duration than many other studies, which may account for the discrepancies of our findings.

Our results highlight that apathy is distinct from other neuropsychiatric syndromes and support the hypothesis that it might be a cognitive disturbance related to cognitive decline in AD. Apathy is composed of an affective, motor, and cognitive dimension. The cognitive component of apathy in $\mathrm{AD}$ could be the most influential. As the cognitive decline in $\mathrm{AD}$ worsened, there was an increasing occurrence of the apathetic syndrome in patients. Further, it was extremely common, occurring in more than half the patients with $\mathrm{AD}$ and more frequently than all the other syndromes, even the affective one. This is in agreement with a previous report that apathy is the most common neuropsychiatric symptom in $\mathrm{AD}^{29}$ which is unrelated to depression in dementia patients. ${ }^{30}$ In addition, the fact that apathy has often been found in conjunction with depression and anxiety may be caused by the subtle differences in apathy and depression that could be misinterpreted. ${ }^{31}$ Our clinicians are experienced in geriatric psychiatry, especially in the evaluation of neuropsychiatric symptoms in $\mathrm{AD}$, and they were careful to accurately diagnose and distinguish depressive and apathetic symptoms during interview with patients and caregivers. If apathy is a cognitive symptom in $\mathrm{AD}$, then it could be linked with frontal and executive dysfunction. Magnetic resonance imaging studies show an association between apathy and medial frontal region structural abnormality in $\mathrm{AD}_{1}^{32}$ and there is neuropathologic evidence of increased neurofibrillary tangles burden in the anterior cingulate cortex of patients with $\mathrm{AD}$ with apathy. ${ }^{33}$ Apathy is highly associated with executive functioning, which is a cognitive domain dependent on the frontal cortex. Together, these findings show that apathy is the most frequent psychiatric symptom in $\mathrm{AD}$, which is related to the disease process and cognitive decline.

The prevalence of syndromes was high; more than three quarters of patients had at least one syndrome, and almost $60 \%$ had one at a clinically significant level. This is compatible with previous reports concerning the prevalence of single behavioral symptoms in dementia. ${ }^{4,8}$ This suggests that the syndrome approach does not reduce the sensitivity for identifying behavioral manifestations in patients with AD. The advantage of considering neuropsychiatric syndromes instead of single symptoms is that the disturbance can be regarded in a more coherent way, particularly in view of specific treatments. The syndrome approach reduces the problems associated with situations in which patients with AD have mild symptoms that have uncertain clinical relevance.

Of the five syndromes, only the affective one did not show an increased occurrence in the most severe forms of cognitive impairment. It is known that depression may be present in the early phases of $\mathrm{AD}{ }^{34}$ Less is known about the other component, anxiety, although research shows a high prevalence of anxiety in patients 
in the prodromal " $\mathrm{MCI}$ " phase of $\mathrm{AD} .^{35}$ It has been suggested that neuropsychiatric symptoms represent functional psychological responses to decreasing global cognitive capacity. ${ }^{4}$ Although mood and memory, the domains compromised both in affective disorders and $\mathrm{AD}$, share a common anatomic bases of the limbic circuits, depression and anxiety may be epiphenomena of the disease rather than direct consequences of neurodegeneration, especially because we found no increase in the occurrence of the affective syndrome with increasing dementia severity.

Our finding that psychiatric syndromes in patients with $\mathrm{AD}$ are consistent with primary psychiatric disorders in $\mathrm{AD}$ supports the idea that the pathologic process of $\mathrm{AD}$ causes cognitive and psychological disturbances. Indeed, positron emission tomography (PET) studies in apathy, depression, and psychoses of patients with $\mathrm{AD}^{33,36,37}$ show a reduction of brain metabolism localized in regions consistent with the observed symptoms. Furthermore, we observed a distinct syndrome of psychosis in our patients with $\mathrm{AD}$, characterized by delusions and hallucinations, which supports previous research suggesting that psychosis is a distinct clinical syndrome in $\mathrm{AD}_{1}^{13,14}$ and that patients with $\mathrm{AD}$ with hallucinations have distinct neuropathologic and clinical characteristics. ${ }^{38}$

One limitation of our study is that principal component analysis provides results that are best suited to the data set on which it is conducted. Thus, the syndromes we have identified might not be representative of the pattern in other populations. For this reason, our syndromes need to be verified in other independent cohorts of patients. Furthermore, because we applied specific exclusion criteria for this study, our results are applicable only to patients with similar characteristics. It is also possible that there may be a certain level of ascertainment bias in our study because we used clinical data. Patients with $\mathrm{AD}$ with neuropsychiatric symptoms might be more likely to seek medical help than patients with $\mathrm{AD}$ without such symptoms. Indeed, research has shown that the time between onset of initial symptoms and first consultation with a physician varies largely between patients, with a long delay to diagnosis in some patients with $\mathrm{AD} \cdot{ }^{19,21-23}$ Furthermore, the cross-sectional design of our study limits the interpretation of the associations between disease severity and neuropsychiatric symptoms, because these factors may also influence the decision to seek medical help. Conversely, a major strength of our study is that we examined a large cohort of patients with $\mathrm{AD}$ who were not treated either for AD or neuropsychiatric disturbances, which may affect the natural history and pattern of behavioral symptoms. ${ }^{12,15,16} \mathrm{Be}-$ cause of the cross-sectional design of the study, we were able to examine the occurrence of neuropsychiatric syndromes at the point of first diagnosis, without the confounding effect of drug interventions.

Our study has relevant clinical and research implications. First, our findings support the use the syndrome approach for the clinical evaluation of neuropsychiatric symptoms in AD. Clinicians should include a comprehensive psychiatric evaluation of patients with $\mathrm{AD}$, which focuses on the co-occurrence of behavioral symptoms and characterizes the distinct syndromes when planning treatment and care strategies. Second, our results have relevance to public health planning. Behavioral disturbances in $\mathrm{AD}$ are associated with institutionalization ${ }^{39}$ and caregiver burden. ${ }^{40}$ Information on the prevalence and pattern of neuropsychiatric syndromes can aid public health planners to predict the care needs of patients and their families. Finally, our findings help to better understand disease mechanisms and pathologic changes in $\mathrm{AD}$ that might be associated with the different syndromes of neuropsychiatric disturbances.

In conclusion, our study supports the use of the syndrome approach for diagnosing neuropsychiatric problems in patients with AD. Clinicians should incorporate a thorough psychiatric and neurologic examination of patients with $\mathrm{AD}$ and consider therapeutic strategies that focus on psychiatric syndromes, rather than specific individual symptoms. Finally, considering the high prevalence and severity of neuropsychiatric syndromes, long-term care plans and health care for patients with $\mathrm{AD}$ should take into account the risk of behavioral symptoms in addition to cognitive and functional decline.

The authors thank all patients and their families for participating in the study. The study was partially supported by Italian Ministry of Health by "Progetti Finalizzati 2004." Supported by the European Research Council under the European Community's Seventh Framework Programme (FP7-PEOPLE-2007-2-1-IEF)/ERC grant agreement number [200913] (to KP). No disclosures to report. 


\section{References}

1. Finkel SI, Costa Silva J, Cohen G, et al: Behavioral and psychological signs and symptoms of dementia: a consensus statement on current knowledge and implication for research and treatment. Int Psychogeriatr 1996; 8(suppl 3):497-500

2. Suh GH, Kim SK: Behavioral and psychological signs and symptoms of dementia (BPSD) in antipsychotic-naive Alzheimer's disease patients. Int Psychogeriatr 2004; 16:337-350

3. Eustace A, Coen R, Walsh C, et al: A longitudinal evaluation of behavioural and psychological symptoms of probable Alzheimer's disease. Int J Geriatr Psychiatry 2002; 17:968-973

4. Engelborghs S, Maertens K, Nagels G, et al: Neuropsychiatric symptoms of dementia: cross-sectional analysis from a prospective, longitudinal Belgian study. Int J Geriatr Psychiatry 2005; 20:1028-1037

5. Spalletta G, Baldinetti F, Buccione I, et al: Cognition and behaviour are independent and heterogeneous dimensions in Alzheimer's disease. J Neurol 2004; 251:688-695

6. Low LF, Brodaty H, Draper B: A study of premorbid personality and behavioural and psychological symptoms of dementia in nursing home residents. Int J Geriatr Psychiatry 2002; 17:779783

7. Aalten P, van Valen E, de Vugt ME, et al: Awareness and behavioral problems in dementia patients: a prospective study. Int Psychogeriatr 2006; 18:3-17

8. Robert PH, Verhey FR, Byrne EJ, et al: Grouping for behavioral and psychological symptoms in dementia: clinical and biological aspects. Consensus paper of the European Alzheimer disease consortium. Eur Psychiatry 2005; 20:490 - 496

9. Hollingworth P, Hamshere ML, Moskvina V, et al: Four components describe behavioral symptoms in 1,120 individuals with late-onset Alzheimer's disease. J Am Geriatr Soc 2006; 54:13481354

10. Frisoni GB, Rozzini L, Gozzetti A, et al: Behavioral syndromes in Alzheimer's disease: description and correlates. Dement Geriatr Cogn Disord 1999; 10:130-138

11. Aalten P, de Vugt ME, Lousberg R, et al: Behavioral problems in dementia: a factor analysis of the Neuropsychiatric Inventory. Dement Geriatr Cogn Disord 2003; 15:99-105

12. Aalten P, Verhey FR, Boziki M, et al: Consistency of neuropsychiatric syndromes across dementias: results from the European Alzheimer Disease Consortium. Part II. Dement Geriatr Cogn Disord 2008; 25:1-8

13. Jeste DV, Finkel SI: Psychosis of Alzheimer's disease and related dementias. Diagnostic criteria for a distinct syndrome. Am J Geriatr Psychiatry 2000; 8:29-34

14. Ropacki SA, Jeste DV: Epidemiology of and risk factors for psychosis of Alzheimer's disease: a review of 55 studies published from 1990 to 2003. Am J Psychiatry 2005; 162:20222030

15. Cummings JL, McRae T, Zhang R; Donepezil-Sertraline Study Group: Effects of donepezil on neuropsychiatric symptoms in patients with dementia and severe behavioral disorders. Am J Geriatr Psychiatry 2006; 14:605-612

16. Wang LY, Shofer JB, Rohde K, et al: Prazosin for the treatment of behavioral symptoms in patients with Alzheimer disease with agitation and aggression. Am J Geriatr Psychiatry 2009; 17:744751

17. McKhann G, Drachman D, Folstein M, et al: Clinical diagnosis of Alzheimer's disease: report on the NINCDS-ADRDA Work Group under the auspices of the Department of Health and Human
Services Task Force on Alzheimer's Disease. Neurology 1984; 34:939-944

18. Folstein M, Folstein S, McHugh P: "Mini-mental state." A practical method for grading the cognitive states of patients for the clinician. J Psychiatr Res 1975; 12:189-198

19. Sheng B, Law CB, Yeung KM: Characteristics and diagnostic profile of patients seeking dementia care in a memory clinic in Hong Kong. Int Psychogeriatr 2009; 21:392- 400

20. McCarten JR, Hemmy LS, Rottunda SJ, et al: Patient age influences recognition of Alzheimer's disease. J Gerontol A Biol Sci Med Sci 2008; 63:625-628

21. Knopman D, Donohue JA, Gutterman EM: Patterns of care in the early stages of Alzheimer's disease: impediments to timely diagnosis. J Am Geriatr Soc 2000; 48:300-304

22. Clark PC, Kutner NG, Goldstein FC, et al: Impediments to timely diagnosis of Alzheimer's disease in African Americans. J Am Geriatr Soc 2005; 53:2012-2017

23. Cattel C, Gambassi G, Sgadari A, et al: Correlates of delayed referral for the diagnosis of dementia in an outpatient population. J Gerontol A Biol Sci Med Sci 2000; 55:M98-M102

24. Lawton MP, Brody EM: Assessment of older people: self-maintaining and instrumental activities of daily living. Gerontologist 1969; 9:179-186

25. Cummings JL, Mega M, Gray K, et al: The Neuropsychiatric Inventory: comprehensive assessment of psychopathology in dementia. Neurology 1994; 44:2308-2314

26. Schneider IS, Tariot PN, Lyketsos CG, et al: National Institute of Mental Health Clinical Antipsychotic Trials of Intervention Effectiveness (CATIE): Alzheimer disease trial methodology. Am J Geriatr Psychiatry 2001; 9:346-360

27. Proitsi P, Hamilton G, Tsolaki M, et al: A Multiple Indicators Multiple Causes (MIMIC) model of Behavioural and Psychological Symptoms in Dementia (BPSD). Neurobiol Aging, 2009 [Epub ahead of print]

28. Moran M, Walsh C, Lynch A, et al: Syndromes of behavioural and psychological symptoms in mild Alzheimer's disease. Int J Geriatr Psychiatry 2004; 19:359-364

29. Landes AM, Sperry SD, Strauss ME: Prevalence of apathy, dysphoria, and depression in relation to dementia severity in Alzheimer's disease. J Neuropsychiatry Clin Neurosci 2005; 17:342-349

30. Starkstein SE, Petracca G, Chemerinski E, et al: Syndromic validity of apathy in Alzheimer's disease. Am J Psychiatry 2001; 158:872-877

31. Tagariello P, Girardi P, Amore M: Depression and apathy in dementia: same syndrome or different constructs? A critical review. Arch Gerontol Geriatr 2009; 49:246-249

32. Apostolova LG, Akopyan GG, Partiali N, et al: Structural correlates of apathy in Alzheimer's disease. Dement Geriatr Cogn Disord 2007; 24:91-97

33. Marshall GA, Fairbanks LA, Tekin S, et al: Neuropathologic correlates of apathy in Alzheimer's disease. Dement Geriatr Cogn Disord 2006; 21:144-147

34. Olin JT, Katz IR, Meyers BS, et al: Provisional diagnostic criteria for depression of Alzheimer disease: rationale and background. Am J Geriatr Psychiatry 2002; 10:129-141

35. Palmer K, Berger AK, Monastero R, et al: Predictors of progression from mild cognitive impairment to Alzheimer disease. Neurology 2007; 68:1596-1602

36. Hirono N, Mori E, Ishii K, et al: Frontal lobe hypometabolism and depression in Alzheimer's disease. Neurology 1998; 50: $380-383$ 
37. Mega MS, Lee L, Dinov ID, et al: Cerebral correlates of psychotic symptoms in Alzheimer's disease. J Neurol Neurosurg Psychiatry 2000; 69:167-171

38. Tsuang D, Larson EB, Bolen E, et al: Visual hallucinations in dementia: a prospective community-based study with autopsy. Am J Geriatr Psychiatry 2009; 17:317-323
39. Tun SM, Murman DL, Long HL, et al: Predictive validity of neuropsychiatric subgroups on nursing home placement and survival in patients with Alzheimer disease. Am J Geriatr Psychiatry 2007; 15:314-327

40. Tun SM, Murman DL, Colenda CC: Concurrent validity of neuropsychiatric subgroups on caregiver burden in Alzheimer disease patients. Am J Geriatr Psychiatry 2008; 16:594-602 